\title{
Incident Mining using Structural Prototypes
}

\author{
Ute Schmid ${ }^{1}$ Martin Hofmann ${ }^{1}$, Florian Bader ${ }^{1}$, Tilmann Häberle ${ }^{2}$, and \\ Thomas Schneider ${ }^{2}$ \\ 1 Faculty Information Systems and Applied Computer Science, University of \\ Bamberg, Germany, < firstname $>$.<lastname $>@$ uni-bamberg.de \\ 2 SAP AG, St. Leon-Rot, Germany, $<$ firstname $>$.<lastname $>@$ sap.com
}

\begin{abstract}
Software and other technical products offered to a mass market have a high demand on support and help desks. A tool for automated classification of incident reports, errors and other customer requests which offers previous (successful) hints or solution procedures could efficiently decrease support costs. We propose an approach to mining incidents and other customer requests for support based on generalising structural prototypes from structured data. Retrieval can then be efficiently realised by matching incoming requests against prototypes. We present an application to incident reports in an SAP business information system. Several variants of structure generalisation algorithms were realised and performance for an example test base was evaluated with promising results.
\end{abstract}

\section{Introduction}

In many business domains, especially for software companies, dealing with user requests for support and service has a growing demand on time and costs. Such user requests might be concerned with lack of information or understanding of installing or using the software, with the need specialised routines for non-standard problems, or with the report of errors and the need of trouble-shooting strategies. While general recommendations for very frequent requests can be collected on a FAQ site, typically many requests have to be dealt with on an individual basis. If support is distributed between many employees, possibly also distributed over different locations, it can be often the case that one support engineer has to deal with a problem which another support engineer has already solved on a previous occasion. A common data base of user requests and how they were handled (successfully) could reduce time and effort for service and support dramatically. Such a data base could even be the backbone for automated support answers for simple standard requests.

Given a data base with support requests and solution routines, the main problem is to provide a suitable similarity measure for retrieval of a suitable solution routine for a new request. In the context of a case-based reasoning approach (Aamodt \& Plaza, 1994), similarity is determined between a new and an already known case. Alternatively, cases can be generalised into prototypes (Rosch, 1983; Zadeh, 1982; Wilson \& Martinez, 1993). In this case, the most similar prototype 
is retrieved and - depending on the application domain - either the associated standard solution can be applied to the new case or a parametrised solution routine can be instantiated in accordance to the new case. Using prototypes can have an advantage over cases for large data bases because retrieval time can be reduced when new cases have only to be matched against the prototypes and not against all cases. Furthermore, prototype theory (Rosch, 1983) mimics a successful human cognitive strategy (Wiese, Konerding, \& Schmid, 2008). A prototype represents the relevant aspects of a set of similar objects or situations while irrelevant details are ignored.

The most prominent approach to similarity in data mining, case-based reasoning and classifier learning is to use feature based measures such as Euclidean distances or other Minkowski metrics (Everitt, Landau, \& Leese, 2001). If data are not given as sets of features but in form of a structured representation e.g., as records or as terms - there are two strategies to obtain a similarity rating available: An obvious approach is to transform the structures into feature sets (Yan, Zhu, Yu, \& Han, 2006; Geibel, Schädler, \& Wysotzki, 2003). This has the advantage that many standard approaches to data-mining and classification can be used. Another possibility is to use specialised approaches to structural similarity such as edit distance (Bunke \& Messmer, 1994) or determining greatest common structures (Messmer \& Bunke, 2000; Plaza, 1995; Estruch, Ferri, Hernández-Orallo, \& Ramírez-Quintana, 2009). Structure-based approaches have the advantage that information contained in the relations between objects is not lost by transformation into features.

Our area of application is concerned with incident reports for the business information software SAP Business ByDesign. In this software, creation and visualisation of incident reports are based on an incident model. Incident reports are created by an incident wizard. The current system state together with all context data (current workspace, current object, UI) and a filled in report mask are saved into an XML document and sent first to a key user and - if he or she cannot solve the problem - sent further to a support engineer at SAP. The reports can be viewed by the support engineer in the SAP support studio software where he or she has different possibilities to analyse the report using a graphical presentation of context data.

The work presented here, is a first exploration of the utility of structure generalisation in this domain. Currently, we work on manually created incident clusters and focus on generalisation and retrieval. In the following, we first present how incidents are represented in form of trees. Afterwards, we introduce our approach to tree generalisation and retrieval. Within the general framework of structure matching and learning we propose different algorithmic realisations. An evaluation of these realisations and a comparison with the inductive logic programming algorithm FolL for a set of sample incidents is presented. We conclude with possible improvements, extensions, and suggestions for application. 


\section{Incident Trees}

Incidents occurring in the context of the SAP Business ByDesign System are represented in a unique form, given as an incident model which is specified as an XML-tree. It contains, for example, information about the software version, the workcenter (the role of the user) and the business object for which the incident occurred. The model is an abstraction of the system's class hierarchy. Incident objects are referred to by a name, their corresponding class is given as a type. The details of the incident model are reported in Bader (2009). The general structure of an incident follows the following form: Each element has a prescribed type which characterises the context information and the content of an incident. For a given incident, an element is instantiated with a name-string. Depending on its type, an element can have zero to a typically small number $n$ of children. An extract of the incident model is given in Figure 1.

An example use case is, that an employee wants to order some office equipment. He or she works from the "home" workcenter (WC(home)) and entered

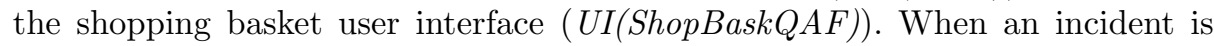
reported in this situation, the business objet "purchase request" (BO(PurReq)) becomes part of the incident context.

The incident model - also called model tree - represents the general structure which is underlying each possible incident report and thereby restricts the form of incident trees.

Definition 1. A model tree $M$ is a tree of fixed size with typed elements $e: \tau$. The type of the element determines the number and types of its child nodes.

Note, that in Figure 1 we write an element $e$ as $\tau$ (name) where name is a variable which can be instantiated by a constant name-string for a given incident.

Definition 2. An incident tree $I$ is an instantiation of the model tree $M$ : Each element $e \in M$ is either mapped to $\epsilon$ (empty element) or a constant name string of type $\tau$. At least one element in I must be unequal $\epsilon$.

Definition 3. To refer to an element $e$ in a tree $T$, we write $e$ if addressing the element only and we write $e\left(T_{1}, \ldots, T_{n}\right)$ if we address the element and its children. A position in a tree $T$ is defined as

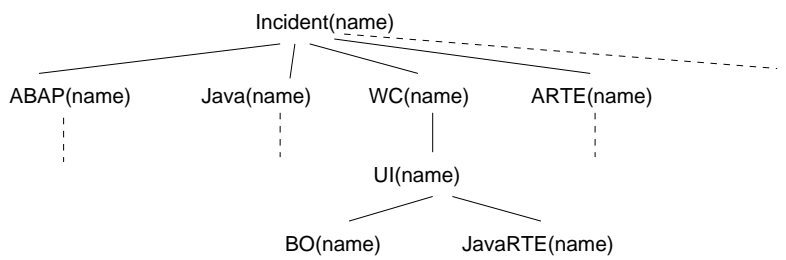

Fig. 1. Extract of the Incident Model 
$-\lambda$ is the root position of $T$,

- if $T=e\left(T_{1}, \ldots T_{n}\right)$ and $u$ is a position in $T_{i}$, then i.u. is a position in $T$.

$T . p=e$ refers to a specific element in T. T.p $=e\left(T_{1}, \ldots, T_{n}\right)$ refers to a specific element and its children.

For better readability we omit types in the definition. In the algorithms presented in the following section, it is guaranteed that mapped elements are of the same type since mapping is guided by a fixed model tree underlying all instance trees.

\section{Tree Generalisation and Retrieval}

For learning of incident prototypes, sets of incidents - called cluster - are generalised with respect to their common structure. In consequence, each cluster is represented by a prototype and new incoming incidents are compared with all prototypes to retrieve the most similar one. In the following we propose three approaches to generalisation and retrieval: Anti-unification of trees as base-line approach and two variants for generating structure-dominance trees. Since we are not concerned with arbitrary trees but with trees based on a unique structure given as model tree, there is no need to rely on general approaches to tree matching (Wang, Zhang, Jeong, \& Shasha, 1994).

\subsection{Anti-Unification of Trees}

Syntactic first-order anti-unification is an approach to generate least generalisations over terms (Plotkin, 1969; Burghardt \& Heinz, 1996). An anti-instance of a set of terms is calculated by traversing them simultaneously and keeping the common structure. If terms start with different symbols, these terms are represented as mappings to variables in the anti-instance. The mappings can be used to recreate the original terms from the anti-instance by transforming them into substitutions.

Since trees and terms are corresponding data structures, this approach can be transferred to incident trees. That is, a cluster prototype is defined as antiinstance of a set of incident trees. Instead of calculating mappings, we introduce an $\epsilon$-element in the anti-instance at the position of mismatched terms.

Algorithm 1. Let $M$ be a model tree, $I=\left\{I_{1}, \ldots, I_{N}\right\}$ a set of incident trees, and $P$ a prototype tree. Syntactic anti-unification of sets of incident trees $\mathrm{au}(\mathrm{p}, \mathrm{P}, \mathrm{M}, \mathrm{I})$ is defined as:

- Initially the prototype is empty: P. $\lambda=\epsilon$.

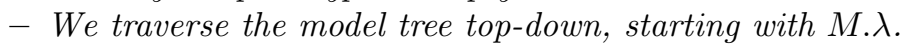

- For the current position $p$ in model tree $M, M . p=e: \tau$

- If for all incident trees in I holds $I_{1} \cdot p=\ldots=I_{N} \cdot p$

then P.p :=e and

if $M . p=e\left(T_{1}, \ldots, T_{n}\right)$ then for all incidents $I_{i}$ do au $\left(p . i, P, M,\left\{T_{1 i}, \ldots, T_{N i}\right\}\right)$. 
- Else P.p $:=\epsilon: \tau$ (an empty element).

An anti-instance of a set of trees corresponds to the intersection of all trees with respect to a model tree (see Figure 2 for an illustration).

For retrieval, the most similar prototype $P_{i}$ for an incoming incident $I_{\text {new }}$ must be determined. This can be realised by anti-unifying $I_{\text {new }}$ with each prototype and partially ordering the anti-instances $A_{P_{i}, I_{n e w}}$ with respect to their subsumption relation (Plaza, 1995).

Definition 4. An incident tree $T$ is said to subsume another incident tree $T^{\prime}$, that is, $T$ is a generalisation of $T^{\prime}\left(T>T^{\prime}\right)$ if $\operatorname{sub}\left(T, T^{\prime}, \lambda\right)=$ true with

$-\operatorname{sub}\left(\epsilon, T^{\prime}, p\right)=$ true

$-\operatorname{sub}\left(T, T^{\prime}, p\right)=$ false if $T . p \neq \epsilon$ and $T . p \neq T^{\prime} . p$

$-\operatorname{sub}\left(T\left(t_{1}, \ldots, t_{n}\right), T^{\prime}\left(t_{1}^{\prime}, \ldots, t_{m}^{\prime}\right), p\right)=$ true if $T \cdot p=T^{\prime} \cdot p$ and $n=m$ and for all $t_{i} \operatorname{sub}\left(t_{i}, t_{i}^{\prime}, p . i\right)$.

\subsection{Structure Dominance Tree Generalisation}

Syntactic anti-unification is not robust with respect to noise. Furthermore, using an identity criterium for element matching is very strict. If, for example, $n-1$ incidents have an identical element at position $p$ and only one incident has a different or empty entry for this element, the prototype at this position is empty. Therefore, we introduce a new approach to prototype learning - structure dominance tree generalisation (SDTG). The basic idea is to collect the number of occurrences of different elements at a position.

Algorithm 2. Let $M$ be a model tree, $I=\left\{I_{1}, \ldots, I_{N}\right\}$ a set of incident trees, and $P$ a prototype tree. Structure dominance tree generalisation $\operatorname{sdtg}(\mathrm{p}$, $\mathrm{P}, \mathrm{M}, \mathrm{I})$ is defined as:

- Initially the prototype is empty: P. $\lambda=\epsilon$.

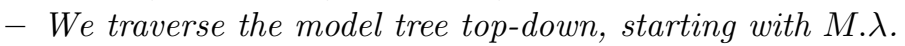

- Until I is empty, for the current position $p$ in model tree $M, M . p=e: \tau$

- If for all incident tree in $I$ holds $I_{1} \cdot p=\ldots=I_{N} \cdot p$ then P.p $:=e: \tau$ and if $M . p=e\left(T_{1}, \ldots, T_{n}\right)$ then for all incidents $I_{i} \operatorname{do} \operatorname{sdtg}\left(p . i, P, M,\left\{T_{1 i}, \ldots, T_{N i}\right\}\right)$.

- Else for each of the $c=\left|\left\{I_{1} . p, \ldots, I_{N} . p\right\}\right|$ different elements create a new node P. $p_{1}, \ldots$, P. $p_{c}$ with P. $p_{i}:=\left[e_{i} / f_{i}\right]: \tau$ as element names $e_{i}$ and their relative frequencies $f_{i}$. Proceed for all new elements with $\operatorname{sdtg}\left(p . i, P, M,\left\{T_{1 i}, \ldots, T_{N i}\right\}\right)$.

While syntactic anti-unification returns the intersection of a set of incident trees as prototype, SDTG returns the union of all incident trees (see Figure 2 for an illustration).

A combination of both approaches can be realised as follows: 


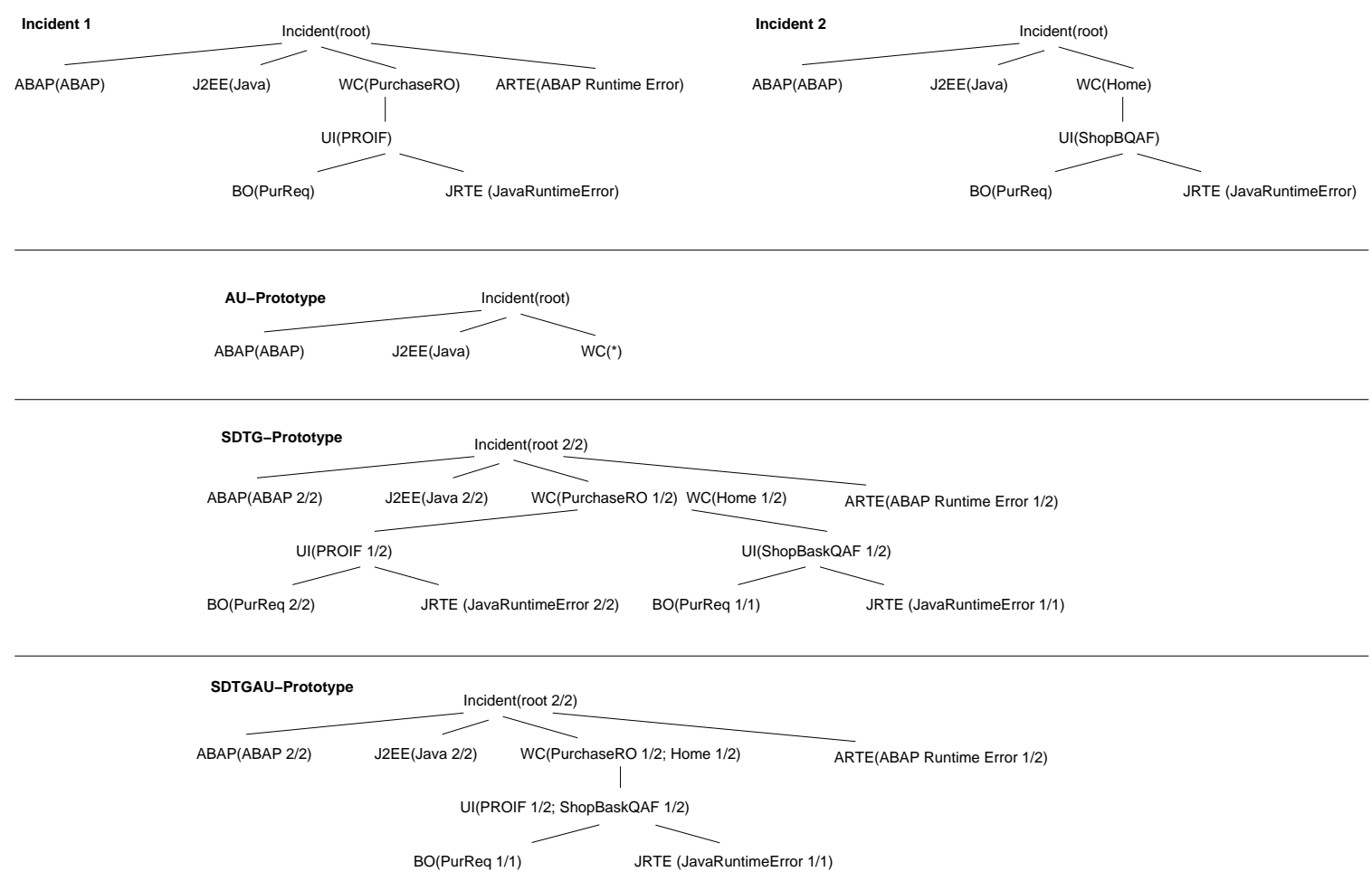

Fig. 2. Illustration of Prototype Generation

Algorithm 3. Let $M$ be a model tree, $I=\left\{I_{1}, \ldots, I_{N}\right\}$ a set of incident trees, and $P$ a prototype tree. Structure dominance tree generalisation with anti-unification $\operatorname{sdtgau}(\mathrm{p}, \mathrm{P}, \mathrm{M}, \mathrm{I})$ is defined as:

- Initially the prototype is empty: P. $\lambda=\epsilon$.

- We traverse the model tree top-down, starting with M. $\lambda$.

- Until I is empty, for the current position $p$ in model tree $M, M . p=e: \tau$

- If for all incident tree in $I$ holds $I_{1} \cdot p=\ldots=I_{N} \cdot p$ then P.p $:=e$ and

if $M . p=e\left(T_{1}, \ldots, T_{n}\right)$ then for all incidents $I_{i}$ do $\operatorname{sdtgau}\left(p . i, P, M,\left\{T_{1 i}, \ldots, T_{N i}\right\}\right)$.

- Else P.p $:=\left[e_{i} / f_{i}\right]: \tau$ becomes a tuple of all occurring elements at position $p$ together with their relative frequencies and we proceed for all children with $\operatorname{sdtgau}\left(p . i, P, M,\left\{T_{1 i}, \ldots, T_{N i}\right\}\right)$.

An illustrative example is given in Figure 2.

Retrieval for SDTG and SDTGAU can be realised using some similarity measure over trees. We explored several measures and it showed, that Manhattan distance is the most robust and reliable measure:

$$
d(I, P)=\sum_{i=1}^{n}\left|f_{P i}-f_{I i}\right| \quad \text { with } \quad f_{P i}=1 .
$$




\section{Empirical Results}

For empirical evaluation of our structural approaches to incident mining, we obtained 57 real example incidents from SAP support which were manually grouped in 11 clusters based on their root cause analysis. One cluster contained three incidents, four clusters four incidents each, one cluster five incidents, four clusters six incidents each and one cluster 9 incidents. The rather low number of instances per cluster is realistic for this application domain. That is, for this domain only approaches to prototype or classifier learning which produce reliable results for small numbers of cases are applicable. The size of incidents varied between 27 and 2646 nodes with an average size of 812 nodes.

In addition to the approaches described above, we used the inductive logic programming algorithm FoIL (Quinlan \& Cameron-Jones, 1995) which is a well established approach to learning from relational data. Since FoIL needs positive and negative examples for its rule induction, for each cluster we used one incident from all other clusters as negative example. Furthermore, for using FoIL the incident model was represented as set of Prolog clauses given as background knowledge.

All evaluations were run on an Intel Core 2 Duo with $2.4 \mathrm{GHz}, 2$ GB DDR3 working memory, 250 GB hard disk space and operation system Mac OS X 10.5.6. Algorithms were realised with Java Sun JDK 1.5.0_16-b06-284. FoIL in version 6 was compiled with GCC 4.01. Since running times for all trials had a very low standard deviation for prototype generation as well as for retrieval, we only give average run times and omit giving standard deviations.

For a first evaluation, for each of the 11 clusters the prototypes were generated over all incidents (see Table 1). Afterwards, each instance was used in the retrieval phase. Times for generation of prototypes and for retrieval were averaged over all runs. In general, times for all approaches were reasonably fast. As was to be expected, anti-unification returned perfect results with the smallest prototypes (168 nodes in average). Foll produced a rather large number of erroneous and ambiguous classifications. This result is mostly due to our unsophisticated approach for presentation of negative examples. Since negative examples are used to specialise rules, typically some care in providing suitable negatives is needed. Both SDTG and SDTGAU returned no perfect, but acceptable results.

Table 1. Base Performance: All instances included in prototype generation, all instances used for retrieval

\begin{tabular}{llllcl}
\hline Method & \multicolumn{4}{c}{ Hits Errors } & Av. Size Generation (sec.) Retrieval (sec.) \\
\hline FOIL & 27 & $18,12^{1}$ & & 0,035 & 0,403 \\
AU & 57 & 0 & 168 & 0,177 & 0,038 \\
SDTG & 53 & 4 & 447 & 0,182 & 0,047 \\
SDTGAU & 55 & 2 & 273 & 0,162 & 0,038 \\
\hline
\end{tabular}

${ }^{1}$ first value: classification error, second value: ambiguous result 
Table 2. Leave-one-out Performance: Three trials with one instance excluded from prototype generation

\begin{tabular}{|c|c|c|c|c|c|}
\hline Method & Hits & Errors & Av. Size & Generation (sec.) & $\overline{\text { Retrieval (sec.) }}$ \\
\hline$\overline{\text { FOIL }}$ & 11 & $8,14^{1}$ & & 0,109 & 0,409 \\
\hline $\mathrm{AU}$ & 29 & 4 & 173 & 0,485 & 0,044 \\
\hline SDTG & 22 & 11 & 387 & 0,456 & 0,049 \\
\hline SDTGAU & 31 & 2 & 264 & 0,419 & 0,047 \\
\hline
\end{tabular}

A more precise evaluation was realised using a leave-one-out approach (see Table 2. Due to the small number of examples, we used three runs for prototype generation disregarding the first, the second and the third incident in each cluster respectively. Again, times are given as averages over all runs. Again, all times for prototype construction and for retrieval are acceptable. Now anti-unification returns the wrong prototype in 4 out of 33 cases which is better than SDTG but slightly worse than SDTGAU.

Finally, we evaluated how our approaches can deal with noisy data. Out of the original 11 clusters, we created 110 new clusters, each containing $n-1$ incidents of an original cluster and one incident of one of the other clusters (see Table 3). As was to be expected, anti-unification breaks down for noisy data. SDTGAU outperformed all other approaches returning only 1 misclassification.

Table 3. Performance on noisy clusters: 110 clusters each containing one miss-placed instance

\begin{tabular}{|c|c|c|c|c|c|}
\hline Method & Hits & Errors & $\mathrm{Av}$ & Size Generation (sec.) & Retrieval (sec.) \\
\hline$\overline{\text { FOIL }}$ & 0 & $0,57^{1}$ & & 0,020 & 7,378 \\
\hline $\mathrm{AU}$ & 4 & 53 & 66 & 0,202 & 0,051 \\
\hline SDTG & 52 & 5 & 619 & 0,227 & 0,102 \\
\hline SDTGAU & 56 & 1 & 410 & 0,188 & 0,069 \\
\hline
\end{tabular}

${ }^{1}$ first value: classification error, second value: ambiguous result

\section{Conclusions and Further Work}

For the given application domain - mining of incident reports characterised by an incident model - we could show first promising results for a set of simple structure-generalisation algorithms. Of course, the number of clusters and incidents used in the evaluation is rather small and a further evaluation using a larger data base should be realised. Using a small set of incidents had the advantage that we had full control over clustering which was done manually by a domain expert. For a large scale performance evaluation, we need to extend our approach to automated clustering as a first step. 
For clustering we again propose to take into account the incident structures. That is, we plan to realise a clustering based on structural similarity between instances (Taskar, Segal, \& Koller, 2001).

The proposed algorithms do not take into account the possibility that a tree element might have more than one child of the same type. A planned extension of the algorithms therefore is, to include tree matching to obtain best matches for arbitrary sets of type-identical nodes.

After extension of our approach to automated clustering, we plan to realise a semi-automated assistance tool for support engineers with SDTGAU as generalisation algorithm: For an incoming incident, a ranked list of retrieved prototypes can be offered. If the engineer accepts one of these prototypes, the new incident is saved in the selected cluster. The support engineer furthermore can edit the prescribed support routines associated with the cluster prototypes. In repeated intervals - after substantial growth of the incident data base - automated clustering and prototype generalisation can be re-done to stratify the data-base. We assume that such a tool might relieve support engineers of repetitive work, considerably heighten efficiency of support and ultimately provide fast and reliable support for the users.

\section{References}

Aamodt, A., \& Plaza, E. (1994). Case-based reasoning: foundational issues, methodological variations, and system approaches. AI Communications, $7(1), 39-59$.

Bader, F. (2009). Model-based classification of incident reports in a business information system. Sn approach to prototype-learning by structure generalisation (in German). Unpublished master's thesis, University of Bamberg.

Bunke, H., \& Messmer, B. T. (1994). Similarity measures for structured representations. In S. Wess, K.-D. Althoff, \& M. Richter (Eds.), Proc. 1st European Workshop on Topics in Case-Based Reasoning (Vol. 837, pp. 106-118). Springer.

Burghardt, J., \& Heinz, B. (1996). Implementing anti-unification modulo equational theory (Vol. 1006; Tech. Rep.). Arbeitspapiere der GMD.

Estruch, V., Ferri, C., Hernández-Orallo, J., \& Ramírez-Quintana, M. (2009). Defining inductive operators using distances over lists. In U.Schmid, E. Kitzelmann, \& R. Plasmeijer (Eds.), Proceedings of the 3rd Workshop on Approaches and Applications of Inductive Programming (AAIP'09) (pp. 41-64). Edinburgh.

Everitt, B. S., Landau, S., \& Leese, M. (2001). Cluster analysis, 4th edition. Wiley.

Geibel, P., Schädler, K., \& Wysotzki, F. (2003). Connectionist construction of prototypes from decision trees for graph classification. Intelligent Data Analysis, 7(2), 125-140. 
Messmer, B. T., \& Bunke, H. (2000). Efficient subgraph isomorphism detection: A decomposition approach. Efficient Subgraph Isomorphism Detection: A Decomposition Approach, 12(2), 307 - 323.

Plaza, E. (1995). Cases as terms: A feature term approach to the structured representation of cases. In Proc. 1st International Conference on CaseBased Reasoning (ICCBR-95) (Vol. 1010, pp. 265-276). Springer.

Plotkin, G. D. (1969). A note on inductive generalization. In Machine intelligence (Vol. 5, pp. 153-163). Edinburgh University Press.

Quinlan, J., \& Cameron-Jones, R. (1995). Induction of logic programs: FOIL and related systems. New Generation Computing, Special Issue on Inductive Logic Programming, 13(3-4), 287-312.

Rosch, E. (1983). Prototype classification and logical classification: The two systems. In E. Scholnick (Ed.), New trends in conceptual representation: Challenges to Piaget's theory? (p. 73-86). Hillsdale, NJ: Lawrence Erlbaum.

Taskar, B., Segal, E., \& Koller, D. (2001). Probabilistic clustering in relational data. In Seventeenth International Joint Conference on Artificial Intelligence (IJCAI-01) (p. 870-887).

Wang, J. T. L., Zhang, K., Jeong, K., \& Shasha, D. (1994). A system for approximate tree matching. IEEE Transactions on Knowledge and Data Engineering, 6(4), 559-571.

Wiese, E., Konerding, U., \& Schmid, U. (2008). Mapping and inference in analogical problem solving - As much as needed or as much as possible? In B. Love, K. McRae, \& V. M. Sloutsky (Eds.), Proceedings of the 30th Annual Conference of the Cognitive Science Sociecty (p. 927-932). Mahwah, NJ: Lawrence Erlbaum.

Wilson, D. R., \& Martinez, T. R. (1993). The potential of prototype styles of generalization. In Proceedings of the Sixth Australian Joint Conference on Artifical Intelligence (AI'93) (pp. 356-361).

Yan, X., Zhu, F., Yu, P. S., \& Han, J. (2006). Feature-based similarity search in graph structures. ACM Transactions on Database Systems, 31 (4), 1418 $-1453$.

Zadeh, L. (1982). A note on prototype theory and fuzzy sets. Cognition, 12, 291-297. 